\title{
Comparisons of neurotrophic effects of mesenchymal stem cells derived from adipose tissue, bone marrow, and cranial bone on chronic spinal cord injury
}

\section{Takashi Otsuka}

Hiroshima University: Hiroshima Daigaku https://orcid.org/0000-0002-7892-441X

\section{Yuyo Maeda}

Hiroshima University: Hiroshima Daigaku

\section{Tomoyuki Kurose}

Hiroshima University: Hiroshima Daigaku

\section{Kei Nakagawa}

Hiroshima University: Hiroshima Daigaku

\section{Takafumi Mitsuhara}

Hiroshima University: Hiroshima Daigaku

\section{Yumi Kawahara}

Space Bio-Laboratories Co., Ltd.

\section{Kaoru Kurisu}

Chugoku Rosai Hospital: Chugoku Rosai Byoin

\section{Louis Yuge ( $\nabla$ ryuge@hiroshima-u.ac.jp )}

Hiroshima University

\section{Research}

Keywords: Mesenchymal stem cells, Chronic spinal cord injury, Cranial bone, Neural crest, Neurotrophic factor, Neural plasticity factor, Motor functional improvement

Posted Date: March 4th, 2021

DOl: https://doi.org/10.21203/rs.3.rs-273086/v1

License: (1) This work is licensed under a Creative Commons Attribution 4.0 International License. Read Full License 


\section{Abstract \\ Background}

Cell-based therapies with mesenchymal stem cells (MSCs) are considered as promising strategies for spinal cord injury (SCl). MSCs have unique characteristics due to difference in the derived tissues. However, relatively few studies have focused on differences in the therapeutic effects of MSCs derived from different tissues. Here, the therapeutic effects of adipose tissue-derived MSCs (aMSCs), bone marrow-derived MSCs (bMSCs), and cranial bone-derived MSCs (cMSCs) on chronic SCI model rats were compared.

\section{Methods}

MSCs were established from adipose tissue, bone marrow, and cranial bone collected. Neurotrophic factor expression of each MSC type was analyzed by real-time PCR. SCI rats were established with the weight-drop method and transplanted intravenously with MSCs at 4 weeks after SCl. Hind-limb motor function was evaluated from before injury to 4 weeks after transplantation. Endogenous neurotrophic factor and neural repair factor expression in spinal cord (SC) tissue were examined by real-time PCR and western blot analyses. Furthermore, the neurotrophic effects (i.e., neurite formation and elongation) of each MSC type were verified by co-culture with NG108-15 neural cells.

\section{Results}

Although there were no differences in the expression levels of cell surface markers and multipotency, expression of Bdnf, Ngf, and Sort1 (Nt-3) was relatively higher in cMSCs. Transplantation of cMSCs improved motor function of chronic $\mathrm{SCl}$ model rats. Although there was no difference in the degree of engraftment of transplanted cells in the injured SC tissue, transplantation of cMSCs enhanced Bdnf, TrkB, and Gap-43 mRNA expression and synaptophysin protein expression in injured SC tissue. In vitro, cMSCs co-cultured with NG108-15 cells promoted neurite formation and elongation.

\section{Conclusion}

As compared with MSCs derived other tissues, cMSCs highly express many neurotrophic factors which improved motor function in chronic $\mathrm{SCl}$ model rats by promoting endogenous neurotrophic and neural plasticity factors. These results suggest the efficacy of cMSCs in cell-based therapy for chronic SCl.

\section{Background}

The main causes of spinal cord injury (SCl) include traffic accidents, tumors, and degenerative diseases. $\mathrm{SCl}$ is a serious burden to healthcare systems because of the large patient population [1] and remaining 
severe dysfunction. The acute phase of $\mathrm{SCl}$ is characterized by contusion, laceration, stretch, compression, or direct massive destruction of the spinal cord (SC). Subsequent second injuries such as inflammation and cytotoxic edema, lead to the apoptosis of neurons at the lesion site [2-5]. The chronic phase of $\mathrm{SCl}$ is characterized by the formation of a glial scar after gliosis. Although the formation of a glial scar border prevents further expansion of tissue damage in the acute phase, glial scar formation in the chronic phase limits for axonal regrowth [6, 7]. However, even under such circumstances, synapse plasticity and axonal sprouting are promoted by various molecules [8]. Thus, it is important to identify the mechanisms that regulate neural regeneration to promote functional recovery after $\mathrm{SCl}$. Although physical rehabilitation [9] and drugs $[10,11]$ have been used to treat SCls, there is currently no effective treatment strategy.

Among the many strategies for the SCl treatment, cell-based therapy is considered one of the most important and promising. Mesenchymal stem cells (MSCs) are especially appealing because of the potential of self-renewal and multi-lineage differentiation, secretion of humoral factors, migratory capacity, and ability to promote endogenous neurogenesis [12-14]. MSCs, which are isolated mainly from the bone marrow [15-17], have been investigate in numerous clinical trials $[15,18]$. Other than the bone marrow, MSCs can be isolated from adipose tissue, dental pulp, and Wharton jelly [19, 20]. Previous studies have identified differences in the characteristics of MSCs derived from various tissue [20-22]. Our group has focused on the cranial bone which originates from the neural crest as a new source of MSCs. Shinagawa et al. showed that cranial bone-derived MSCs (cMSCs) have a higher neurogenic potential than bone marrow-derived MSCs (bMSCs) [23], whereas Abiko et al. found differences in the therapeutic effects between bMSCs and cMSCs with the use of an ischemic stroke model [24]. Thus, unique characteristics of MSCs derived from various tissues may have different therapeutic effects due to their unique characteristics. Although it is necessary to choose optimal MSCs for each central nervous system (CNS) disease and stage of illness, the timing of treatment with MSCs for SCl was mainly in the acute or subacute phase in previous studies [25-27]. In addition, relatively few reports have investigated differences in the therapeutic effects of MSCs derived from various tissued on chronic SCl. Hence, further studies are needed to investigate the effects and underlying mechanisms of various MSCs for the treatment for chronic $\mathrm{SCl}$ is an important issue. Therefore, the present study aimed to compare the neurotrophic effects of adipose tissue-derived MSCs (aMSCs), bMSCs, and cMSCs in chronic SCl model rats.

\section{Methods}

\section{Isolation and culture of aMSCs, bMSCs and cMSCs}

For isolation of MSCs, we collected abdominal adipose tissue, femur, tibia, and cranial bone from adult female Sprague-Dawley (SD) rats (body weight, 250-300g). The adipose tissues collected were digested with Collagenase-A type AFA (560 U/mg; Worthington Biochemical Corporation, Lakewood, NJ, USA) and filtered. The extracts were centrifuged and then seeded into tissue culture dishes (Sumitomo Bakelite Co., Tokyo, Japan) containing culture medium, consisting of low-glucose Dulbecco's modified Eagle's medium 
(DMEM; Sigma-Aldrich Corporation, St Louis, MO, USA), supplemented with $10 \%$ fetal bovine serum (FBS; Thermo Fisher Scientific, Waltham, MA, USA), penicillin $(100 \mathrm{U} / \mathrm{mL})$, and streptomycin $(100 \mu \mathrm{g} / \mathrm{mL}$, both from Sigma-Aldrich). The adhesion of aMSCs was detected the next day after seeding. In the present study, bMSCs and cMSCs were established as described previously [24]. Briefly, bone marrow was collected from the femur and tibia, filtered, and the resulting cells were seeded into culture dishes. Once the cells had adhered to the bottom of the culture dishes at about 5-7 days, non-adherent cells were eliminated by changing the culture medium. The adherent cells were used as bMSCs. After removing muscle, periosteum, and dura matter, cranial bones were seeded into culture dishes. The adhesion of cMSCs was detected at 5-7days after seeding. Cells were maintained at $37^{\circ} \mathrm{C}$ under an atmosphere of $5 \%$ $\mathrm{CO}_{2} / 95 \%$ air and the culture medium was changed every 3 days after the detection of cell adhesion. The aMSC, bMSC, and cMSC collected were passaged to more than $80 \%$ confluence.

\section{Flow cytometry analysis}

aMSCs, bMSCs, and cMSCs at passage 3 were collected with TrypLE ${ }^{\text {TM }}$ Select Cell Dissociation Solution (Thermo Fisher Scientific), centrifuged at $1500 \times g$ for $5 \mathrm{~min}$, and then resuspended in phosphatebuffered saline (PBS) $(n=3)$. Aliquots containing $1 \times 10^{5}$ cells were incubated with fluorochromeconjugated fluorescein isothiocyanate (FITC) or phycoerythrin (PE)-conjugated antibodies (Abs) against rat CD29 (562154; BD Biosciences, San Jose, CA, USA), CD34 (sc-7324; Santa Cruz Biotechnology Inc., Dallas, TX, USA), CD44 (203906; Biolegend, San Diego, CA, USA), CD45 (554877; BD Biosciences), and CD90 (554898; BD Biosciences). PE-conjugated mouse immunoglobulin (Ig)G1 and FITC-conjugated mouse IgG1 (400108 and 400112; both from Biolegend) were used as an isotype control. FITCconjugated antibody (Ab) against CD44 and PE-conjugated Abs against CD29, CD90, were used as MSC markers. FITC-conjugated $\mathrm{Ab}$ against CD45 and PE-conjugated $\mathrm{Ab}$ against CD34 were used as hematopoietic markers. Flow cytometry was conducted using the BD FACSVerse ${ }^{\mathrm{TM}}$ Flow Cytometer and data acquisition and analyses were performed using BD FACSuite ${ }^{T M}$ software (both, BD Biosciences).

\section{Multi-lineage cell differentiation}

aMSCs, bMSCs, and cMSCs at passage 3 or 4 were used for differentiation into osteoblasts, adipocytes, or neurons. To induce osteogenic differentiation, cells were cultured in Mesenchymal Stem Cell Osteogenic Differentiation Medium (PromoCell GmbH, Heidelberg, Germany) for 21 days, which changes to the medium every 3 or 4 days. To confirm calcium deposition, the cells were stained with alizarin red S solution (Sigma-Aldrich). To induce adipogenic differentiation, cells were cultured in Mesenchymal Stem Cell Adipogenic Differentiation Medium (PromoCell) for 14 days, which change to the medium was changed every 3 or 4 days. Afterward, the cells were finally stained with oil red 0 solution (Wako Pure Chemical Industries, Osaka, Japan) to confirm the formation of lipid droplets. To induce neural differentiation, cells were cultured under modified neural differentiation conditions with the use of neural conditioning medium and neural differentiation medium, as described in previous reports $[23,24]$. MSCs were cultured in neural conditioning medium, which consisted of DMEM: Nutrient Mixture F-12 (Invitrogen Corporation, Carlsbad, CA, USA), 1\% FBS, basic fibroblast growth factor (100 ng/mL; 
PeproTech Inc., Rocky Hill NJ, USA), penicillin (100 U/mL), and streptomycin (100 $\mu \mathrm{g} / \mathrm{mL})$. After culturing maintained in neural conditioning medium for 3 days, the medium was changed to the neural differentiation medium composed of neural conditioning medium with forskolin ( $10 \mu \mathrm{M}$; Sigma-Aldrich) for 7 days. The differentiation medium was changed every 3 or 4 days. After the neural differentiation process, the cultured cells were fixed in $4 \%(\mathrm{w} / \mathrm{v})$ paraformaldehyde and incubated with anti- $\beta$-tubulin III mouse monoclonal Ab (T8578; 1:200, Sigma-Aldrich) as the primary $A b$ overnight at $4^{\circ} \mathrm{C}$, followed by Alexa Fluor 488-conjugated anti-mouse IgG Ab (H+L) (A11029; dilution, 1:500, Molecular Probes Europe BV Co., Leiden, Netherlands) as the fluorescent secondary Ab at room temperature. The nuclei were stained with 4',6-diamidino-2-phenylindole dihydrochloride (DAPI) (71-03-00; dilution, 1:800, Kirkegaard \& Perry Laboratories, Gaithersburg, MD, USA). The stained cells were examined under a multifunctional microscope (BZ-9000, KEYENCE Co., Osaka, Japan).

\section{Reverse transcription and real-time polymerase chain reaction (PCR) of MSCs}

At confluence, the cultured cells after reached confluency were collected in PBS. Total RNA was extracted using the NucleoSpin ${ }^{\circledR}$ RNA kit (MACHEREY-NAGEL GmbH \& Co. KG, Düren, Germany), in accordance with the manufacturer's protocol. The purified RNA was reverse transcribed into complementary deoxyribonucleic acid (cDNA) using the ReverTra Ace- $\mathrm{a}^{-{ }^{\mathrm{TM}} \mathrm{M}}$ reverse transcription kit (Toyobo Co., Ltd., Osaka, Japan). Using cDNA as a template, real-time PCR was performed with the 7900 HT Real-Time PCR system (Applied Biosystems, Carlsbad, CA, USA), in accordance with the manufacturer's instructions. Real-time PCR amplification and analyses of rat brain-derived neurotrophic factor $(B d n f)$, glial cell-derived neurotrophic factor ( $G d n f)$, fibroblast growth factor 2 ( $F g f 2)$, nerve growth factor (Ngf), and sortilin 1, which is also known as neurotrophin 3 (Sort1/Nt-3), were performed using the FastStart $^{\text {TM }}$ Universal Probe Master Mix (Roche, Basel, Switzerland) and TaqMan ${ }^{\text {TM }}$ Gene Expression Assays (Applied Biosystems). Actin beta (Actb) was used as the loading control to normalize the relative quantity of the specific messenger ribonucleic acid (mRNA) in each sample. The TaqMan Gene Expression Assays used in this study are listed in Table 1.

\section{Table 1. TaqMan Assay primers used for real-time PCR of mRNA expression of each MSC type.}




\begin{tabular}{ccc}
\hline Gene symbol & Gene name & Assay ID \\
\hline Actb & Actin beta & Rn00667869_m1 \\
Bdnf & Brain-derived neurotrophic factor & Rn02531967_s1 \\
Fgf2 & Fibroblast growth factor 2 & Rn00570809_m1 \\
Gdnf & Glial cell-derived neurotrophic factor & Rn00569510_m1 \\
Ngf & Nerve growth factor & Rn01533872_m1 \\
Sort1 (Nt-3) & Sortilin 1 (Neurotrophin 3) & Rn01521847_m1 \\
\hline
\end{tabular}

\section{Surgical procedure}

Adult female SD rats (body weight, 250-300 g) were used to construct a SCI model with the weightdropping method $[26,28]$. The rats were anesthetized and a midline linear incision was made over the thoracic (Th) 9-11 spinous processes. After exposing the laminae of Th9-11, laminectomy was carried out at Th10. An impactor rod was set on the surface of the SC at Th10 and a cylindrical brass weight (10 g) was dropped onto the impactor to create a SC contusion was made with a force of $50 \mathrm{~g} / \mathrm{cm}$. Following the contusion, the skin was sutured to close the lesion. After the surgical procedure, the rats were administered antibiotics for 5 days postoperatively to prevent infection. The bladders of the $\mathrm{SCl}$ rats were compressed manually twice daily until autonomic bladder function had recovered sufficiently. After the $\mathrm{SCl}$, the rats also received passive joint motion exercises daily to prevent contracture of after the hindlimb joints.

\section{Experimental groups and cell transplantation}

$\mathrm{SCl}$ rats were assigned to one of the following four treatment groups ( $\mathrm{n}=10$ each): only PBS group; aMSCs group; bMSCs group; cMSCs group. Rats in the aMSC, bMSC, and CMSC groups were injected intravenously with $1.0 \times 10^{6} \mathrm{MSCs}$ in $300 \mu \mathrm{L}$ of PBS at 4week post the SCl. To identify the transplanted MSCs in the recipient SCl rats, the MSCs were labeled with PKH26 (Sigma-Aldrich) just before transplantation.

\section{Motor functional assessment of SCl model rats}

The inclined plane test and the Basso-Beattie-Bresnahan locomotor rating scale (BBB scale) were used to evaluate hind limb function. The BBB scale is a 22-point scale that systematically follows the recovery of hind-limb function, and ranges from a score of 0 , indicative of no observed movement of the hind-limb, to a score of 21, normal ambulation [29]. The inclined plane test assesses the maximum angle 
at which the rats can hold a position for $5 \mathrm{~s}$ on an inclined plane [30]. Motor function was assessed just before the $\mathrm{SCl}$, on day 1 post the $\mathrm{SCl}$, and weekly until sacrifice at week 8 post the $\mathrm{SCl}$.

\section{Detection of transplanted cells in SC}

To detect the PKH26-labeled transplanted cells, SCs were collected and cryoprotected. Rats were euthanized by deep anesthesia and transcardial perfusion of $4 \%$ paraformaldehyde. The fixed SCs that were removed from the vertebral columns, soaked in $30 \%$ sucrose solution, embedded in Tissue-Tek O.C.T compound (Sakura Finetechnical Co., Tokyo, Japan), and frozen in liquid nitrogen. Afterward, the spinal columns were sliced at a thickness of $10 \mu \mathrm{m}$ with a cryostat and the sections were encapsulated by VECTASHIELD Mounting Medium with DAPI (H-1200, Vector Laboratories, Burlingame, CA, USA) to stain the cell nuclei. The encapsulated sections were examined under a multifunctional microscope (KEYENCE Co.).

\section{real-time PCR analysis of SC tissue}

Five rats from each group were sacrificed 4 weeks after MSC transplantation. RNA from the SCs was isolated with the ISOGEN RNA extraction reagent (Nippon Gene, Tokyo, Japan) by centrifugation and reverse transcribed into cDNA, as described above. Real-time PCR analyses of rat $B d n f$, tropomyosin receptor kinase B (TrkB), growth-associated protein 43 (Gap-43), and synaptophysin (Syn) were performed using TaqMan Gene Expression Assays. Actb was used as the loading control to normalize the relative quantity of the specific mRNA in each sample. The TaqMan Gene Expression Assays used in this study are listed in Table 2.

Table 2. TaqMan Assay primers used for real-time PCR of mRNA expression in SC tissue.

\begin{tabular}{ccc}
\hline Gene symbol & Gene name & Assay ID \\
\hline Actb & Actin beta & Rn00667869_m1 \\
Bdnf & Brain-derived neurotrophic factor & Rn02531967_s1 \\
Gap-43 & Growth associated protein 43 & Rn01474579_m1 \\
Syn & Synaptophysin & Rn00561986_m1 \\
TrkB & Tropomyosin receptor kinase B & Rn01441749_m1 \\
\hline
\end{tabular}

\section{Western blotting analysis}

After RNA extraction, total protein was extracted from the remaining lysate and concentrations were measured with the Bio-Rad protein assay (Bio-Rad Laboratories, Hercules, CA, USA). Proteins (20 $\mu \mathrm{g}$ per lane) were loaded into the lanes of a $15 \%$ polyacrylamide gel, separated by electrophoresis, and 
transferred to nitrocellulose membranes (HybondTM-ECL; GE Healthcare, Little Chalfont, UK). Membranes were blocked with a blocking buffer $(20 \mathrm{mM}$ Tris- $\mathrm{HCl}$ [pH 7.4], $137 \mathrm{mM} \mathrm{NaCl}, 0.1 \%$ Tween-20, $1 \%$ bovine serum albumin) for $60 \mathrm{~min}$ at room temperature and then incubated overnight at $4^{\circ} \mathrm{C}$ with primary $\mathrm{Abs}$ against BDNF (GTX132621; dilution 1:2000; GeneTex Inc., Irvine, CA, USA), GAP-43 (GTX127937; dilution 1:10000; GeneTex), and SYN (ab32127; dilution 1:5000; Abcam, Cambridge, UK). After washing with Trisbuffered saline with Tween 20, the membranes were incubated with horseradish peroxidase (HRP)conjugated anti-rabbit IgG (PI-1000; dilution 1:2000; Vector Laboratories) as the secondary Ab. The immunoreaction was visualized using either the SuperSignal ${ }^{\circledR}$ West Pico Chemiluminescent Substrate (Pierce, Rockford, IL, USA). Images were acquired with the VersaDoc ${ }^{\mathrm{Tm}}$ imaging system (Bio-Rad). The membranes were then stripped with a solution of $62.5 \mathrm{mM}$ Tris- $\mathrm{HCl}, 2 \%$ sodium dodecyl sulfate, and 100 mM 2-mercaptoethanol ( $\mathrm{pH}$ 6.8), and labeled with HRP-conjugated anti-Actb Ab (A3854; Sigma-Aldrich). The expression levels of the target proteins were quantified by measuring the band densities using Image J software (version 1.53; National Institutes of Health, Bethesda, MD, USA). Actb was used as the loading control for data normalization.

\section{Immunohistochemical analysis}

For immunohistochemical analyses, the fixed SCs were embedded in paraffin using standard methods. Paraffin blocks were sliced into $10-\mu \mathrm{m}$-thick sections, which were rehydrated using xylene and ethanol solutions. After treatment with antigen retrieval solution at $98^{\circ} \mathrm{C}$ for $15 \mathrm{~min}$, nonspecific binding was blocked with $1 \%$ bovine serum albumin in PBS for $60 \mathrm{~min}$. Afterward, the sections were incubated overnight with rabbit anti-SYN Ab (dilution, 1:500; Abcam) at $4^{\circ} \mathrm{C}$, followed by FITC-conjugated goat antirabbit IgG (F9887; dilution, 1:100; Sigma-Aldrich) for $60 \mathrm{~min}$ at room temperature. Then, the stained sections were incubated overnight with mouse anti-Tuj1 Ab (GTX631836, 1:250, GeneTex) at $4^{\circ} \mathrm{C}$, followed by Rhodamine Red-X-AffiniPure Fab Fragment Donkey Anti-Mouse IgG (715-297-003; dilution, 1:100; Jackson ImmunoResearch Europe Ltd., Suffolk, UK) for $60 \mathrm{~min}$ at room temperature. The stained sections were encapsulated by VECTASHIELD Mounting Medium with DAPI (Vector Laboratories) to stain the cell nuclei. The encapsulated sections were examined under a multifunctional microscope (KEYENCE Co.).

\section{NG108-15 neural cell culture}

NG108-15 neuroblastoma / glioma hybrid cells (ECACC, Porton Down, UK), which were used as a model of motor neurons, were seeded into culture dishes and cultured in high-glucose DMEM (SigmaAldrich), supplemented with 10\% FBS (Thermo Fisher Scientific), penicillin $(100 \mathrm{U} / \mathrm{mL})$, streptomycin $(100$ $\mu \mathrm{g} / \mathrm{mL}$, both from Sigma-Aldrich), and HAT supplement (Thermo Fisher Scientific) at $37^{\circ} \mathrm{C}$ under an atmosphere of $5 \% \mathrm{CO}_{2} / 95 \%$ air.

\section{Co-culture of MSCs and NG108-15 neural cells}

aMSCs, bMSCs, and cMSCs at passage 3 were seeded at 20,000 cells per $\mathrm{cm}^{2}$ in the wells of sixwell plates (Sumitomo Bakelite). As a negative control (no co-culture), only the MSC growth medium was 
added to the well. After $24 \mathrm{~h}$, the NG108-15 cells formed a monolayer at a density of 2,000 cells and the co-culture was continued in DMEM/HAM's F12 (Wako Pure Chemical Industries), supplemented with 1\% FBS (Thermo Fisher Scientific), penicillin $(100 \mathrm{U} / \mathrm{mL})$, streptomycin $(100 \mu \mathrm{g} / \mathrm{mL}$, both from SigmaAldrich) for an additional $48 \mathrm{~h}$. Afterward, the cells were fixed in $4 \%(\mathrm{w} / \mathrm{v})$ paraformaldehyde. NG108-15 neurite outgrowth was assessed by fluorescent immunocytochemical analysis. After blocking with $1 \%$ bovine serum albumin for $60 \mathrm{~min}$ at room temperature, the cells were incubated overnight with mouse anti-Tuj $1 \mathrm{Ab}$ (dilution, 1:1000; GeneTex) incubated at $4{ }^{\circ} \mathrm{C}$ overnight followed by Alexa Fluor 488conjugated anti-mouse IgG Ab $(\mathrm{H}+\mathrm{L})$ (dilution, 1:500; Molecular Probes Europe BV) as the fluorescent secondary Ab for 60 min at room temperature. DAPI (dilution, 1:800; Kirkegaard \& Perry Laboratories) was used to stain the cell nuclei. The stained cells were examined under a multifunctional microscope (KEYENCE Co.) and neurite analysis was performed using ImageJ software with the NeuronJ plugin [31]. The following neurite parameters were assessed: (1) the percentage of neurite-like processes; (2) the number of neurites expressed per cell; (3) the average neurite length $(\mu \mathrm{m})$; and (4) the longest neurite length in each group $(\mu \mathrm{m})$. At least 100 neuronal cells from each co-culture were examined. The results are reported as the mean of six independent experiments for each co-culture.

\section{Statistical analysis}

Statistical analyses were performed using JMP software from SAS (version 14; SAS Institute, Cary, NC, USA). Comparisons among groups were performed by one-way analysis of variance (ANOVA) with the post-hoc Tukey's honestly significant difference (HSD) test. Motor functional analysis was performed by two-way ANOVA with the post-hoc Tukey's HSD test. A probability $(p$-) value of $<0.05$ was considered statistically significant.

\section{Results}

\section{Characteristics of MSCs from different tissues}

Flow cytometry was performed to detect surface markers of the aMSCs, bMSCs, and cMSCs. All MSCs were positive for CD29, CD44, and CD90, which are known as MSC positive markers, whereas negative for CD34 and CD45, which are known as MSC negative markers. The expression trends of the immune phenotype were similar for each MSC type (Table 3). The potential of the MSCs to differentiate into osteoblasts, adipocytes and neurons was investigated. After differentiation, the aMSCs, bMSCs, and cMSCs were positively stained with Arizarin Red-S staining, Oil Red-O staining, and anti- $\beta$-tubulin III Ab (Fig. 1A-C). 
Table 3

Surface marker expression of MSCs from various tissues.

\begin{tabular}{|llll|}
\hline MSC positive markers (\%) & aMSCs & bMSCs & cMSCs \\
\hline CD29 & $99.87 \pm 0.12$ & $98.70 \pm 1.02$ & $99.83 \pm 0.17$ \\
\hline CD44 & $99.77 \pm 0.26$ & $99.30 \pm 0.85$ & $99.93 \pm 0.05$ \\
\hline CD90 & $99.23 \pm 0.88$ & $95.87 \pm 1.90$ & $96.00 \pm 3.19$ \\
\hline MSC negative markers (\%) & & & \\
\hline CD34 & $0.19 \pm 0.05$ & $1.70 \pm 0.82$ & $0.75 \pm 0.45$ \\
\hline CD45 & $0.08 \pm 0.04$ & $1.36 \pm 1.42$ & $0.11 \pm 0.11$ \\
\hline
\end{tabular}

The data are presented as the mean + standard deviation (SD) of three independent experiments. aMSCs, adipose tissue-derived MSCs; bMSCs, bone marrow-derived MSCs; cMSCs, cranial bone-derived MSCs; SD, standard deviation.

\section{Neurotrophic factor expression profiles of aMSCs, bMSCs, and cMSCs}

The results of one-way ANOVA identified neurotrophic factors, with significant differences among the MSCs at the mRNA level $(p<0.05)$. Post-hoc analyses showed that $B d n f$ expression was significantly higher in bMSCs $(p<0.05)$ and cMSCs $(p<0.01)$ than in aMSCs (Fig. 2A). Furthermore, Ngf and Sort1 (Nt3) expression was significantly higher in cMSCs than in aMSCs and bMSCs (Ngf: both, $p<0.001$; Sort1: both, $p<0.05$ ) (Fig. 2B, C). On the other hand, Gdnf expression was significantly higher in aMSCs than in bMSCs $(p<0.05)$ (Fig. 2D) and Fgf2 expression was significantly higher in aMSCs than in bMSCs and cMSCs $(p<0.01)$ (Fig. 2E).

\section{Motor functional improvements of chronic SCI rats}

The BBB score and inclined plane test were used to evaluate functional improvement after the $\mathrm{SCl}$ by MSC transplantation. At $24 \mathrm{~h}$ post $\mathrm{SCl}$, most rats showed acute flaccid paralysis with no spontaneous movement of the hind limbs. From day 7 post the SCl, spontaneous coarse movement of the hind limbs was observed, including gradual recovery. However, there was almost no improvement from day 21 post the SCl. MSCs were transplanted at day 28 post the $\mathrm{SCl}$ and were evaluated continuously over a period of 28 days after transplantation. The results of two-way (10 time points $\times 4$ groups) ANOVA revealed significant differences among the groups $(p<0.001)$. Post-hoc analyses were performed at each time point to compare differences among the groups. Based on the BBB score, functional improvements than in the cMSC group were superior to those of the PBS group from day 49 post the $\mathrm{SCl}(p<0.05)$ (Fig. 3A). The results of the inclined plane test demonstrated that rats in the cMSC group were able to maintain a significantly higher angle than in the PBS group from day 42 post the $\mathrm{SCl}$ (days 42 and 56: $p<0.01$; day 
$49, p<0.05)$ (Fig. 3B). Collectively, these results indicate that the greatest functional improvement after the $\mathrm{SCl}$ was obtained with cMSCs.

Localization of transplanted cells in SC

There were no fluorescent cells in the spinal gray matter of the PBS group (Fig. 4A). On the other hand, $\mathrm{PKH} 26$-labeled aMSCs, bMSCs, and cMSCs injected intravenously were detected in the spinal gray matter of each group at day 28 post transplantation (Fig. 4B-D).

Effects of transplantation of different MSCs on neurotrophic and neural plasticity factor expression in s $S C$ tissue

To clarify the effects of transplanted MSCs in SC tissue, mRNA expression levels of $B d n f$, a receptor of $B D N F$, and neuronal plasticity factors were evaluated. The results of one-way ANOVA revealed significant differences in the mRNA expression levels of $B d n f, T r k B$, and Gap-43 in among the different groups ( $p<$ 0.05). The results of post-hoc analyses showed that $B d n f$ and $G a p-43$ expression levels were significantly higher in the cMSC group than those in the PBS and aMSC groups $(p<0.05)$ (Fig. 5A, C). TrkB expression was also significantly higher in the cMSC group than in the PBS group $(p<0.05)$ (Fig. 5B). On the other hand, there were no significant differences in Syn expression levels among the groups (Fig. 5D).

The protein expression levels of BDNF, GAP-43 and SYN were also evaluated. The results of one-way ANOVA revealed significant differences in the protein expression levels of BDNF and SYN among the different groups $(p<0.05)$. The results of post-hoc analysis showed that SYN expression was significantly higher in the cMSC group than in the PBS group $(p<0.05)$ (Fig. $6 \mathrm{C})$, whereas BDNF expression tended to be higher in the CMSC group, but not significantly ( $p=0.0644$ vs. the PBS group; $p=$ 0.0728 vs. the aMSC group; $p=0.0812$ vs. the bMSC group) (Fig. $6 \mathrm{~A}$ ). On the other hand, there were no significant differences in GAP-43 expression among the groups (Fig. 6B).

Next, the localization of SYN was investigated, as SC tissue showed significant changes in protein expression levels. In the motor neurons located in the anterior horn of the SC stained with Tuj1, SYN was confirmed in the contoured part of the neurons in each group. In the PBS group, SYN was sparsely present on the neurons, whereas the SYN content was relatively high in the contoured part of the neurons in each group transplanted with MSCs (Fig. 7A-D). Especially in the cMSC group, the staining intensity of SYN was strong over the entire contour of the neuron (Fig. 7D).

\section{Neurotrophic effects of different MSCs in vitro}

To compare the neurotrophic effects of aMSCs, bMSCs, and cMSCs on neurite outgrowth, different MSCs were co-cultured with NG108-15 cells. After $48 \mathrm{~h}$ of co-culture, the NG108-15 cells were immunostained and the following quantitative parameters were evaluated (Fig. 8A): (1) the percentage of neurite-like processes; (2) the number of neurites expressed per cell; (3) the average neurite length ( $\mu \mathrm{m})$; and (4) the longest neurite length in each group $(\mu \mathrm{m})$. One-way ANOVA revealed significant differences in all 
parameters among the groups $(p<0.05)$ and post-hoc analyses showed that the values of all parameters were significantly greater for NG108-15 neural cells co-cultured with cMSCs $(p<0.05)$ than those of NG108-15 neural cells cultured alone (control). Moreover, the percentage $(71.80 \%)$ of neurite-like processes was greater for co-cultured with cMSCs than that of the control $(47.12 \% ; p<0.01)$ and those co-cultured with bMSCs (58.47\%; $p<0.05)$ (Fig. 8B). Regarding the number of neurites expressed per cell, co-cultured with cMSCs had more neurites than the control in comparison with control (1.62 vs. 0.92/cell, respectively, $p<0.05$ ) (Fig. $8 \mathrm{C}$ ). Regarding the average neurite length, co-cultured with cMSCs had longer neurites than the control ( $58.61 \mu \mathrm{m}$ vs. $45.42 \mu \mathrm{m}$, respectively, $p<0.05)$ (Fig. 8D). Likewise, longest neurite length was greater for co-cultured with cMSCs than for the control $(408.32 \mu \mathrm{m}$ vs. $205.68 \mu \mathrm{m}$, respectively, $p<0.05$ ) (Fig. 8E).

\section{Discussion}

The aim of the present study was to investigate the neurotrophic effects of transplanted MSCs derived from adipose tissue, bone marrow, and cranial bone on functional improvement after SCl.

The results of gene expression analyses revealed differences in the expression patterns of the neurotrophic factors among the MSCs derived from different tissues. In cMSCs, Bdnf, Ngf, and Sort1 (Nt3) expression levels were higher than in aMSCs and bMSCs. On the other hand, Gdnf and Fgf2 expression levels were higher in aMSCs than in bMSCs and cMSCs. Previous studies have reported that the expression levels of neural crest-related genes are stronger in cMSCs than in bMSCs [23, 24]. Sakai et al. observed that dental pulp-derived MSCs (dpMSCs) originating from the same neural crest as the cranial bone have higher expression of BDNF and NT-3 than bMSCs [32]. In addition, Mead et al. demonstrated that dpMSCs have high expression levels of BDNF, NGF, and NT-3, whereas aMSCs have higher expression levels of FGF2 than bMSCs and dpMSCs [33]. The trends in the present study were consistent with previous reports. Thus, the high expression levels of Bdnf, Ngf, and Nt-3 in cMSCs were considered to be related to the origin of the neural crest, which differs from mesoderm germ layers originating from other tissues.

The transplantation effects of MSCs derived from different tissues on motor function were investigated in rat models of chronic SCl. In the cMSC group, the motor function score improved significantly from 2 or 3 weeks after transplantation, but the changes were negligible in the other groups, suggesting that the neurotrophic factors of cMSCs can improve motor function after a SCl.

The results of SC tissue analyses showed that BDNF and GAP-43 mRNA expression and SYN protein expression were significantly higher in the cMSC group than in the PBS and aMSCs groups, with a similar tendency in BDNF protein expression. Furthermore, the intensity of SYN immunostaining of the contours of the motor neurons in the anterior horn of the gray matter was weak in the PBS group, but strong in the cMSC group. In the CNS, BDNF plays important roles in neurogenesis such by promoting synaptic plasticity, myelination, and axonal growth [34-36]. Previous studies have reported that MSCs enhanced endogenous BDNF expression in neural tissue [37-40], suggesting that this effect may be mediated by 
humoral factors released by MSCs [41]. NGF has been reported to increase BDNF expression in neurons via the TrkA/ERK/CREB pathway [42], and Wang et al. demonstrated that transplantation of NT-3expressing MSCs enhances BDNF expression in the injured SC [43]. Therefore, the neurotrophic factors produced by cMSCs may have contributed to the increased expression of endogenous Bdnf in the cMSC group.

GAP-43 is a neuron-specific protein that is abundant in axon growth cones [44]. In the injured SC, GAP-43 promotes axon regeneration, new axon germination, and neurite outgrowth [45]. The SYN protein is abundant in presynaptic vesicles and is an important factor reflecting synaptogenesis and plasticity [46]. GAP-43 was reportedly involved in the synaptic plasticity and nerve repair of a SCI model [47], and fluctuations in SYN expression in injured spinal motor neurons have been suggested as important indicators associated with the loss and recovery of motor function [48, 49]. As changes in GAP-43 and SYN expression levels in the injured SC have significant effects on improving motor function, an increase in these factors in the cMSC group may have contributed to improved motor function. In previous studies, BDNF enhanced GAP-43 and SYN expression in neurons through the Trk receptor and subsequent ERK/CREB signaling $[50,51]$. The promotion of GAP-43 and SYN expression may be mediated by endogenous BDNF of SC tissue that is increased by CMSC transplantation.

It has been confirmed that MSCs are present in the gray matter of the SC tissue after transplantation. Rooney et al. showed that transplanted MSCs survived in the SC for long periods without differentiation and continued to supply neurotrophic factors [52]. Therefore, transplanted MSCs may have direct effects on neurons. Based on these previous reports, in vitro neurite outgrowth experiments using co-culture were conducted to examine the neurotrophic effects on neural cells. Co-culturing with MSCs derived different tissues to assess the tendency of neurite formation showed that cMSCs significantly promoted neurotrophic effects. Previous studies have shown that neurite formation and elongation are mediated by neurotrophic factors such as BDNF, NGF, and GDNF [40, 53-55]. As expected, co-culture with MSCs expressing these factors promoted neurite growth. In particular, cMSCs were more effective than other MSCs because of the greater number of factors and the higher expression levels of factors beneficial to nerve growth.

The results of the present study showed that CMSCs can promote functional recovery in the chronic phases of CNS diseases, suggesting that such effects are mediated by neurotrophic factors. The expected roles of MSCs in developing treatment strategies for $\mathrm{SCl}$, such as suppressing inflammatory responses and apoptosis in the acute phase, and reducing glial scar formation and promoting nerve growth after the subacute phase, vary depending on the stage. In addition, previous studies reported that multiple doses of MSCs produced a more remarkable effect [56, 57]. As these reports focused on MSCs derived from a single tissue, it is important to select MSCs that are most suitable for a particular stage. Hence, and in the future studies are warranted to identify an appropriate combination of MSCs for administration at each stage of $\mathrm{SCl}$.

\section{Conclusions}


In conclusion, the results of the present study showed that cMSCs express an abundance of neurotrophic factors as compared with MSCs derived from other tissues. Further, cMSCs contributed to functional recovery in rat models of chronic SCl by promoting endogenous neurotrophic and neural plasticity factors expression. Even though further studies of cMSCs for the treatment of other diseases and various stages of illness are needed, the results of this study confirmed the efficacy of cMSCs in cell-based therapy for chronic SCl.

\section{Abbreviations}

Ab: antibody

Actb: actin beta

aMSCs: adipose tissue-derived mesenchymal stem cells

ANOVA: analysis of variance

BBB score: Basso-Beattie-Bresnahan locomotor rating score

BDNF: brain-derived neurotrophic factor

bMSCs: bone marrow-derived mesenchymal stem cells

cDNA: complementary deoxyribonucleic acid

cMSCs: cranial bone-derived mesenchymal stem cells

CREB: cyclic adenosine monophosphate response element binding protein

DAPI: 4',6-diamidino-2-phenylindole dihydrochloride

DMEM: Dulbecco's modified Eagle's medium

dpMSCs: dental pulp-derived mesenchymal stem cells

ERK: extracellular signal-regulated kinase

FACS: fluorescence-activated cell sorting

FBS: fetal bovine serum

FGF2: fibroblast growth factor 2

FITC: fluorescein isothiocyanate

GAP-43: growth associated protein 43 
GDNF: glial cell-derived neurotrophic factor

HRP: horseradish peroxidase

mRNA: messenger ribonucleic acid

MSCs: mesenchymal stem cells

NGF: nerve growth factor

PBS: phosphate-buffered saline

PCR: polymerase chain reaction

SC: spinal cord

SCl: spinal cord injury

SD: standard deviation

Sort1 (Nt-3): sortilin 1 (neurotrophin 3)

SYN: Synaptophysin

TrkA: tropomyosin receptor kinase A

TrkB: tropomyosin receptor kinase B

\section{Declarations}

\section{Ethics approval and consent to participate}

All experiments involving the use of animals were conducted in compliance with the guidelines and regulations of the National Institutes of Health, the Animal Testing Committee of Hiroshima University, and the Animal Testing Facility of the Hiroshima University Natural Science Support Center.

Consent for publication

Not applicable.

\section{Availability of data and materials}

All the datasets used and/or analyzed during this study are available from the corresponding authors on reasonable request. 
$\mathrm{LY}$ is a director and shareholder of Space Bio-Laboratories Co., Ltd. (SBL) and YK is a president and shareholder of SBL. The interest conflicts of this research have been approved by the Conflict of Interest Management Committee. By regularly reporting research progress to the Conflicts of Interest Management Committee, we will maintain fairness regarding the interests of this research. All other authors have no personal financial or institutional interest in any of the drugs, materials, or devices described in this article and declare that they have no competing interests.

\section{Funding}

This work was supported in part by Grants-in-Aid for Scientific Research from the Japan Society for the Promotion of Science (JSPS KAKENHI grant no. 18K10709).

\section{Authors' contributions}

TO participated in the design of the study, carried out the in vitro and in vivo experiments, analyzed and interpreted the data, and drafted the manuscript. YM participated in the study design and coordination, carried out the in vivo experiments, and analyzed the data. TK participated in the design of the study, carried out the in vitro and in vivo experiments, and analyzed and interpreted the data. KN participated in the design of the study, analyzed and interpreted the data, and performed the statistical analyses.TM participated in the in vivo experiments, analyzed and interpreted the data, and secured financial support. YK participated in the design of the study and analyzed and interpreted the data. KK participated in the coordination, helped to draft, the final version of the manuscript, and secured financial support. LY conceived the study, participated in its design and coordination, analyzed and interpreted the data, helped to draft the final version of the manuscript, and secured financial support. All authors read and approved the final version of the manuscript for publication.

\section{Acknowledgements}

The authors thank Kiyoharu Shimizu (Department of Neurosurgery, Graduate School of Biomedical and Health Sciences, Hiroshima University) for his support. This work was carried out, in part, at the Analysis Center of Life Sciences, Natural Science Center for Basic Research and Development, Hiroshima University.

\section{References}

1. Cofano F, Boido M, Monticelli M, Zenga F, Ducati A, Vercelli A, et al. Mesenchymal Stem Cells for Spinal Cord Injury: Current Options, Limitations, and Future of Cell Therapy. Int J Mol Sci. 2019;20:2698.

2. Blight AR. Miracles and molecules-progress in spinal cord repair. Nat Neurosci. 2002;5 Suppl:10514.

3. Rowland JW, Hawryluk GW, Kwon B, Fehlings MG. Current status of acute spinal cord injury pathophysiology and emerging therapies: promise on the horizon. Neurosurg Focus. 2008;25:E2. 
4. Garcia E, Aguilar-Cevallos J, Silva-Garcia R, Ibarra A. Cytokine and Growth Factor Activation In Vivo and In Vitro after Spinal Cord Injury. Mediators Inflamm. 2016;2016:9476020.

5. Hayta E, Elden H. Acute spinal cord injury: A review of pathophysiology and potential of non-steroidal anti-inflammatory drugs for pharmacological intervention. J Chem Neuroanat. 2018;87:25-31.

6. Yuan YM, He C. The glial scar in spinal cord injury and repair. Neurosci Bull. 2013;29:421-35.

7. Orr MB, Gensel JC. Spinal Cord Injury Scarring and Inflammation: Therapies Targeting Glial and Inflammatory Responses. Neurotherapeutics. 2018;15:541-53.

8. Bradbury EJ, Burnside ER. Moving beyond the glial scar for spinal cord repair. Nat Commun. 2019;10:3879.

9. van den Brand R, Heutschi J, Barraud Q, DiGiovanna J, Bartholdi K, Huerlimann M, et al. Restoring voluntary control of locomotion after paralyzing spinal cord injury. Science. 2012;336:1182-5.

10. Lima R, Monteiro S, Lopes JP, Barradas P, Vasconcelos NL, Gomes ED, et al. Systemic Interleukin-4 Administration after Spinal Cord Injury Modulates Inflammation and Promotes Neuroprotection. Pharmaceuticals (Basel). 2017;10:83.

11. Martins BC, Torres BBJ, de Oliveira KM, Lavor MS, Osório CM, Fukushima FB, et al. Association of riluzole and dantrolene improves significant recovery after acute spinal cord injury in rats. Spine J. 2018;18:532-9.

12. Jeong $\mathrm{CH}$, Kim SM, Lim JY, Ryu CH, Jun JA, Jeun SS. Mesenchymal stem cells expressing brainderived neurotrophic factor enhance endogenous neurogenesis in an ischemic stroke model. BioMed Res Int. 2014;2014:129145.

13. Leibacher J, Henschler R. Biodistribution, migration and homing of systemically applied mesenchymal stem/stromal cells. Stem Cell Res Ther. 2016;7:7.

14. Feng Y, Ju Y, Cui J, Wang L. Bone marrow stromal cells promote neuromotor functional recovery, via upregulation of neurotrophic factors and synapse proteins following traumatic brain injury in rats. Mol Med Rep. 2017;16:654-60.

15. Honmou O, Houkin K, Matsunaga T, Niitsu Y, Ishiai S, Onodera R, et al. Intravenous administration of auto serum-expanded autologous mesenchymal stem cells in stroke. Brain. 2011;134:1790-807.

16. Sammali E, Alia C, Vegliante G, Colombo V, Giordano N, Pischiutta F, et al. Intravenous infusion of human bone marrow mesenchymal stromal cells promotes functional recovery and neuroplasticity after ischemic stroke in mice. Sci Rep. 2017;7:6962.

17. Nagahama H, Nakazaki M, Sasaki M, Kataoka-Sasaki Y, Namioka T, Namioka A, et al. Preservation of interhemispheric cortical connections through corpus callosum following intravenous infusion of mesenchymal stem cells in a rat model of cerebral infarction. Brain Res. 2018;1695:37-44.

18. Vahidy FS, Haque ME, Rahbar MH, Zhu H, Rowan P, Aisiku IP, et al. Intravenous Bone Marrow Mononuclear Cells for Acute Ischemic Stroke: Safety, Feasibility, and Effect Size from a Phase I Clinical Trial. Stem Cells. 2019;37:1481-91. 
19. Jin HJ, Bae YK, Kim M, Kwon SJ, Jeon HB, Choi SJ, et al. Comparative analysis of human mesenchymal stem cells from bone marrow, adipose tissue, and umbilical cord blood as sources of cell therapy. Int J Mol Sci. 2013;14:17986-8001.

20. Wang Q, Yang Q, Wang Z, Tong H, Ma L, Zhang Y, et al. Comparative analysis of human mesenchymal stem cells from fetal-bone marrow, adipose tissue, and Warton's jelly as sources of cell immunomodulatory therapy. Hum Vaccin Immunother. 2016;12:85-96.

21. Heo JS, Choi Y, Kim HS, Kim HO. Comparison of molecular profiles of human mesenchymal stem cells derived from bone marrow, umbilical cord blood, placenta and adipose tissue. Int J Mol Med. 2016;37:115-25.

22. Du WJ, Chi Y, Yang ZX, Li ZJ, Cui JJ, Song BQ, et al. Heterogeneity of proangiogenic features in mesenchymal stem cells derived from bone marrow, adipose tissue, umbilical cord, and placenta. Stem Cell Res Ther. 2016;7:163.

23. Shinagawa K, Mitsuhara T, Okazaki T, Takeda M, Yamaguchi S, Magaki T, et al. The characteristics of human cranial bone marrow mesenchymal stem cells. Neurosci Lett. 2015;606:161-6.

24. Abiko M, Mitsuhara T, Okazaki T, Imura T, Nakagawa K, Otsuka T, et al. Rat Cranial Bone-Derived Mesenchymal Stem Cell Transplantation Promotes Functional Recovery in Ischemic Stroke Model Rats. Stem Cells Dev. 2018;27:1053-61.

25. Pal R, Gopinath C, Rao NM, Banerjee P, Krishnamoorthy V, Venkataramana NK, et al. Functional recovery after transplantation of bone marrow-derived human mesenchymal stromal cells in a rat model of spinal cord injury. Cytotherapy. 2010;12:792-806.

26. Mitsuhara T, Takeda M, Yamaguchi S, Manabe T, Matsumoto M, Kawahara Y, et al. Simulated microgravity facilitates cell migration and neuroprotection after bone marrow stromal cell transplantation in spinal cord injury. Stem Cell Res Ther. 2013;4:35.

27. Hakim R, Covacu R, Zachariadis V, Frostell A, Sankavaram SR, Brundin L, et al. Mesenchymal stem cells transplanted into spinal cord injury adopt immune cell-like characteristics. Stem Cell Res Ther. 2019;10:115.

28. Basso DM, Beattie MS, Bresnahan JC. Graded histological and locomotor outcomes after spinal cord contusion using the NYU weight-drop device versus transection. Exp Neurol. 1996;139:244-56.

29. Basso DM, Beattie MS, Bresnahan JC. A sensitive and reliable locomotor rating scale for open field testing in rats. J Neurotrauma. 1995;12:1-21.

30. Rivlin AS, Tator $\mathrm{CH}$. Objective clinical assessment of motor function after experimental spinal cord injury in the rat. J Neurosurg. 1977;47:577-81.

31. Meijering E, Jacob M, Sarria JC, Steiner P, Hirling H, Unser M. Design and validation of a tool for neurite tracing and analysis in fluorescence microscopy images. Cytometry A. 2004;58:167-76.

32. Sakai K, Yamamoto A, Matsubara K, Nakamura S, Naruse M, Yamagata M, Sakamoto K, et al. Human dental pulp-derived stem cells promote locomotor recovery after complete transection of the rat spinal cord by multiple neuro-regenerative mechanisms. J Clin Invest. 2012;122:80-90. 
33. Mead B, Logan A, Berry M, Leadbeater W, Scheven BA. Paracrine-Mediated Neuroprotection and Neuritogenesis of Axotomised Retinal Ganglion Cells by Human Dental Pulp Stem Cells: Comparison with Human Bone Marrow and Adipose-Derived Mesenchymal Stem Cells. PLoS One. 2014;9:e109305.

34. Kovalchuk Y, Holthoff K, Konnerth A. Neurotrophin action on a rapid timescale. Curr Opin Neurobiol. 2004;14:558-63.

35. Tuinstra HM, Aviles MO, Shin S, Holland SJ, Zelivyanskaya ML, Fast AG, et al. Multifunctional, multichannel bridges that deliver neurotrophin encoding lentivirus for regeneration following spinal cord injury. Biomaterials. 2012;33:1618-26.

36. Weishaupt N, Blesch A, Fouad K. BDNF: The career of a multifaceted neurotrophin in spinal cord injury. Exp Neurol. 2012;238:254-64.

37. Keefe KM, Sheikh IS, Smith GM. Targeting Neurotrophins to Specific Populations of Neurons: NGF, BDNF, and NT-3 and Their Relevance for Treatment of Spinal Cord Injury. Int J Mol Sci. 2017;18:548.

38. Bao X, Wei J, Feng M, Lu S, Li G, Dou W, et al. Transplantation of human bone marrow-derived mesenchymal stem cells promotes behavioral recovery and endogenous neurogenesis after cerebral ischemia in rats. Brain Res. 2011;1367:103-13.

39. Gutiérrez-Fernández M, Rodríguez-Frutos B, Ramos-Cejudo J, Teresa Vallejo-Cremades M, Fuentes B, Cerdán S, et al. Effects of intravenous administration of allogenic bone marrow- and adipose tissuederived mesenchymal stem cells on functional recovery and brain repair markers in experimental ischemic stroke. Stem Cell Res Ther. 2013;4:11.

40. He X, Jiang L, Dan QQ, Lv Q, Hu Y, Liu J, et al. Bone marrow stromal cells promote neuroplasticity of cerebral ischemic rats via a phosphorylated CRMP2-mediated mechanism. Behav Brain Res. 2017;320:494-503.

41. Asgari Taei A, Dargahi L, Nasoohi S, Hassanzadeh G, Kadivar M, Farahmandfar M. The conditioned medium of human embryonic stem cell-derived mesenchymal stem cells alleviates neurological deficits and improves synaptic recovery in experimental stroke. J Cell Physiol. 2021;236:1967-79.

42. Wang X, Cong P, Liu Y, Tao S, Chen Q, Wang J, et al. Neuritogenic effect of sea cucumber glucocerebrosides on NGF-induced PC12 cells via activation of the TrkA/CREB/BDNF signalling pathway. J Funct Foods. 2018;46:175-84.

43. Wang LJ, Zhang RP, Li JD. Transplantation of neurotrophin-3-expressing bone mesenchymal stem cells improves recovery in a rat model of spinal cord injury. Acta Neurochir. 2014;156:1409-18.

44. Goslin K, Schreyer DJ, Skene JH, Banker G. Development of neuronal polarity: GAP-43 distinguishes axonal from dendritic growth cones. Nature. 1988;336:672-4.

45. Hoang TX, Nieto JH, Havton LA. Regenerating supernumerary axons are cholinergic and emerge from both autonomic and motor neurons in the rat spinal cord. Neuroscience. 2005;136:417-23.

46. Wiedenmann B, Franke WW. Identification and localization of synaptophysin, an integral membrane glycoprotein of Mr 38,000 characteristic of presynaptic vesicles. Cell. 1985;41:1017-28. 
47. Gerin CG, Madueke IC, Perkins T, Hill S, Smith K, Haley B, Allen SA, et al. Combination strategies for repair, plasticity, and regeneration using regulation of gene expression during the chronic phase after spinal cord injury. Synapse. 2011;65:1255-81.

48. Sasaki S, Maruyama S. Decreased synaptophysin immunoreactivity of the anterior horns in motor neuron disease. Acta Neuropathol. 1994;87:125-8.

49. Li GL, Farooque M, Isaksson J, Olsson Y. Changes in synapses and axons demonstrated by synaptophysin immunohistochemistry following spinal cord compression trauma in the rat and mouse. Biomed Environ Sci. 2004;17:281-90.

50. Ma J, Zhang Z, Su Y, Kang L, Geng D, Wang Y, et al. Magnetic stimulation modulates structural synaptic plasticity and regulates BDNF-TrkB signal pathway in cultured hippocampal neurons. Neurochem Int. 2013;62:84-91.

51. Li X, Wu Q, Xie C, Wang C, Wang Q, Dong C, et al. Blocking of BDNF-TrkB signaling inhibits the promotion effect of neurological function recovery after treadmill training in rats with spinal cord injury. Spinal Cord. 2019;57:65-74.

52. Rooney GE, McMahon SS, Ritter T, Garcia Y, Moran C, Madigan NN, et al. Neurotrophic FactorExpressing Mesenchymal Stem Cells Survive Transplantation into the Contused Spinal Cord Without Differentiating into Neural Cells. Tissue Eng Part A. 2009;15:3049-59.

53. Kalbermatten DF, Schaakxs D, Kingham PJ, Wiberg M. Neurotrophic activity of human adipose stem cells isolated from deep and superficial layers of abdominal fat. Cell Tissue Res. 2011;344:251-60.

54. di Summa PG, Kalbermatten DF, Raffoul W, Terenghi G, Kingham PJ. Extracellular Matrix Molecules Enhance the Neurotrophic Effect of Schwann Cell-Like Differentiated Adipose-Derived Stem Cells and Increase Cell Survival Under Stress Conditions. Tissue Eng Part A. 2013;19:368-79.

55. Tomita K, Madura T, Sakai Y, Yano K, Terenghi G, Hosokawa K. Glial differentiation of human adipose-derived stem cells: Implications for cell-based transplantation therapy. Neuroscience. 2013;236:55-65.

56. Yang C, Wang G, Ma F, Yu B, Chen F, Yang J, et al. Repeated injections of human umbilical cord blood-derived mesenchymal stem cells significantly promotes functional recovery in rabbits with spinal cord injury of two noncontinuous segments. Stem Cell Res Ther. 2018;9:136.

57. Krupa P, Vackova I, Ruzicka J, Zaviskova K, Dubisova J, Koci Z, et al. The Effect of Human Mesenchymal Stem Cells Derived from Wharton's Jelly in Spinal Cord Injury Treatment Is DoseDependent and Can Be Facilitated by Repeated Application. Int J Mol Sci. 2018;19:1503.

\section{Figures}




\section{aMSCs}
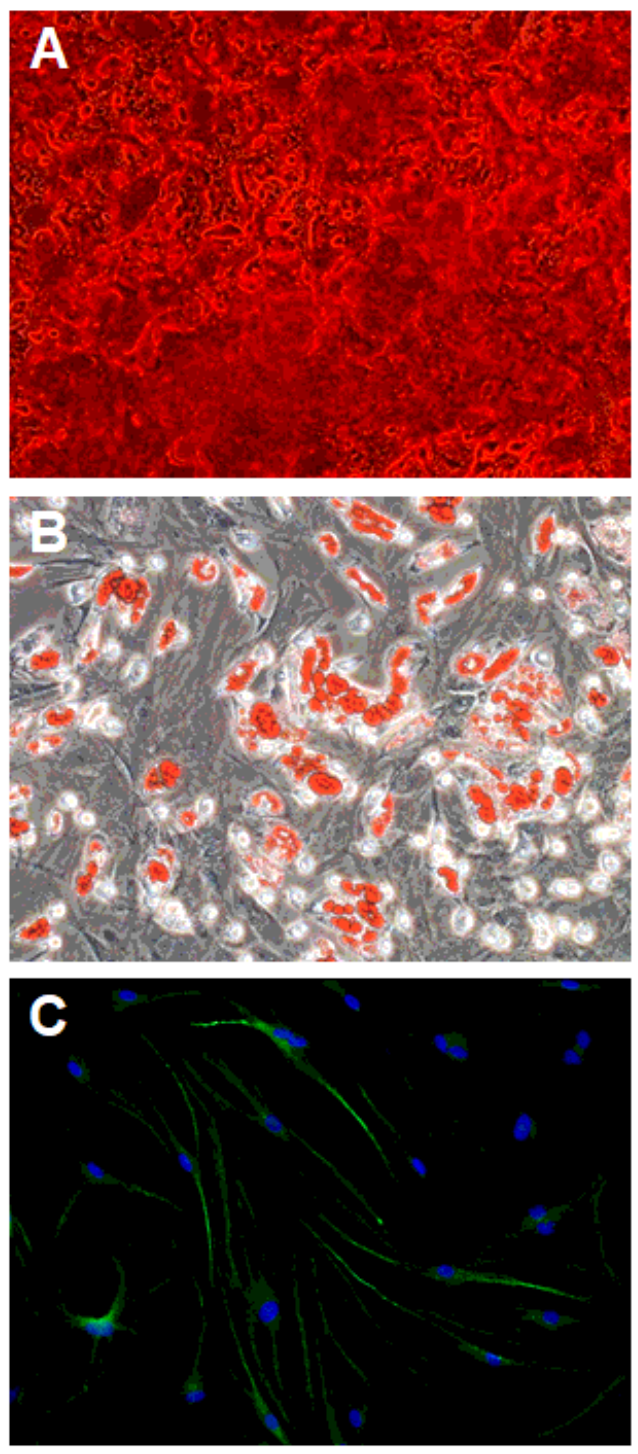

bMSCs
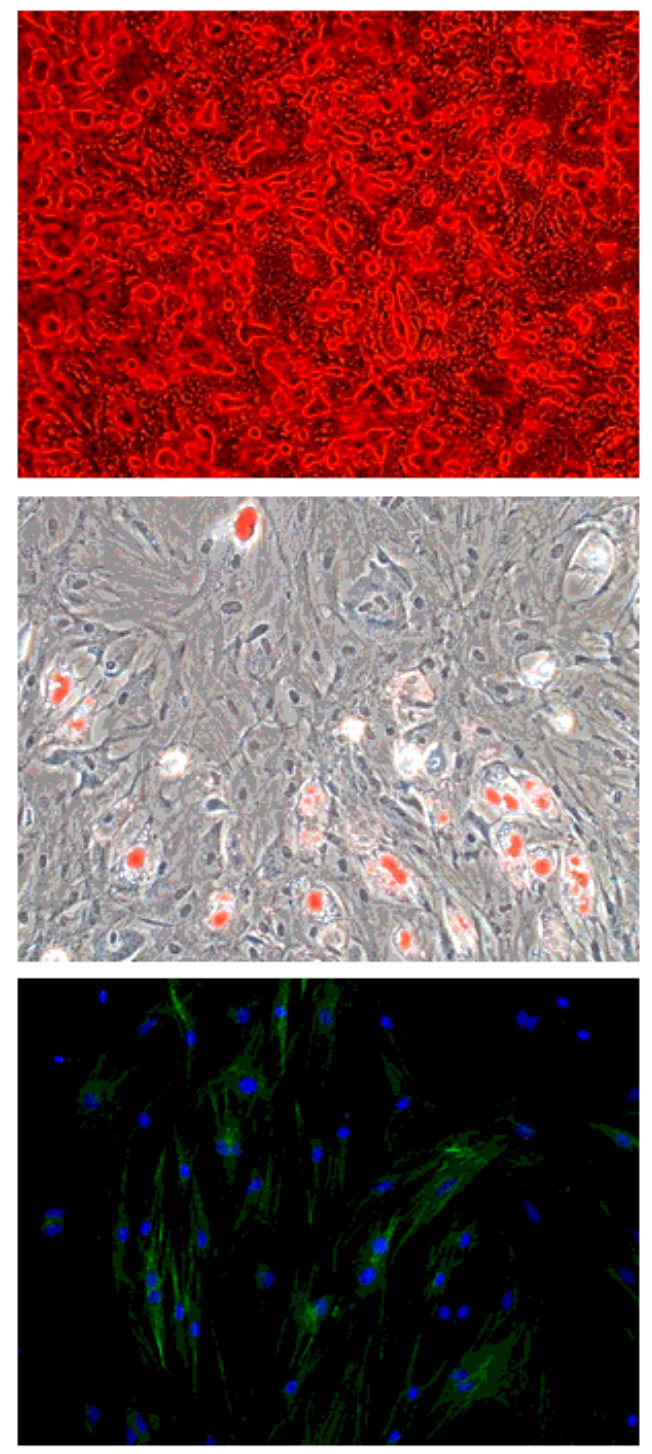

cMSCs
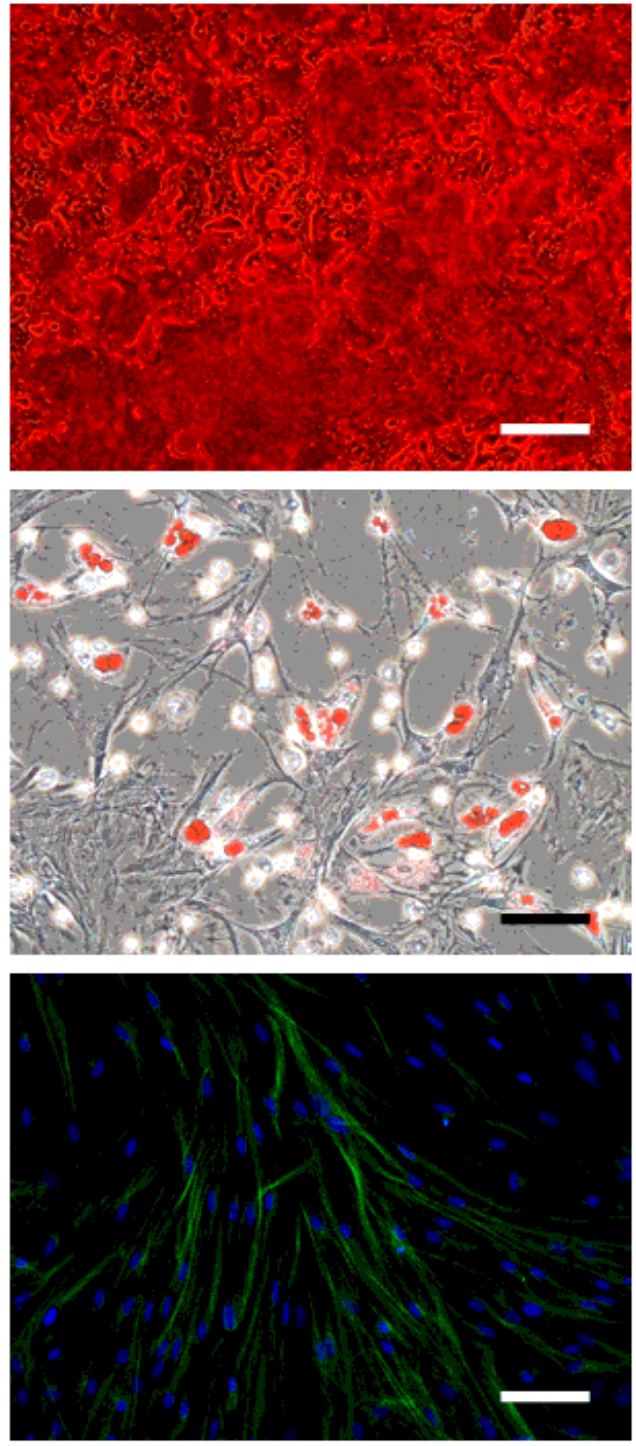

\section{Figure 1}

Specific staining of multi-lineage cell differentiation of aMSCs, bMSCs, and cMSCs. Alizarin red S staining after osteogenic differentiation of aMSCs, bMSCs, and cMSCs (A). Oil red 0 staining after adipogenic differentiation of each MSC type (B). Immunostaining ( $\beta$-tubulin III) after neural differentiation of each MSC type (C). Scale bars $=50 \mu \mathrm{m}$. MSCs, mesenchymal stem cells. 
A

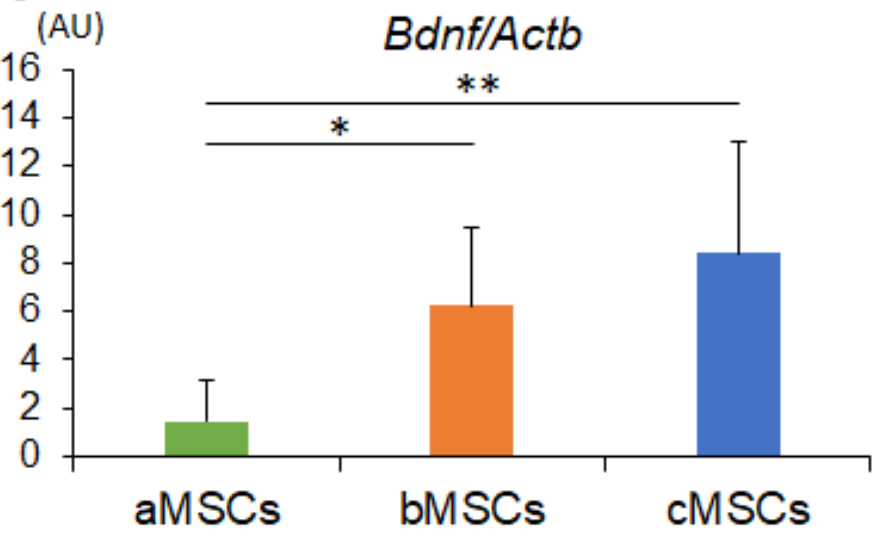

C

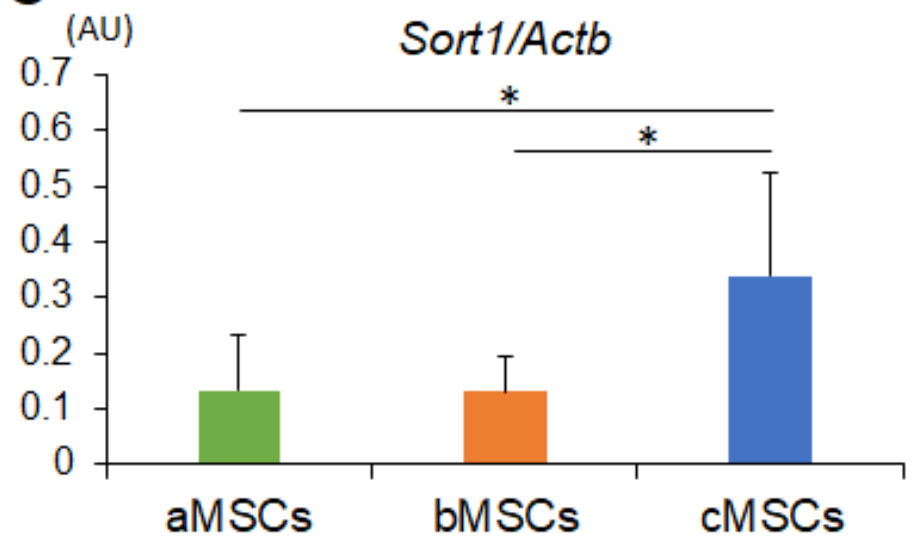

B

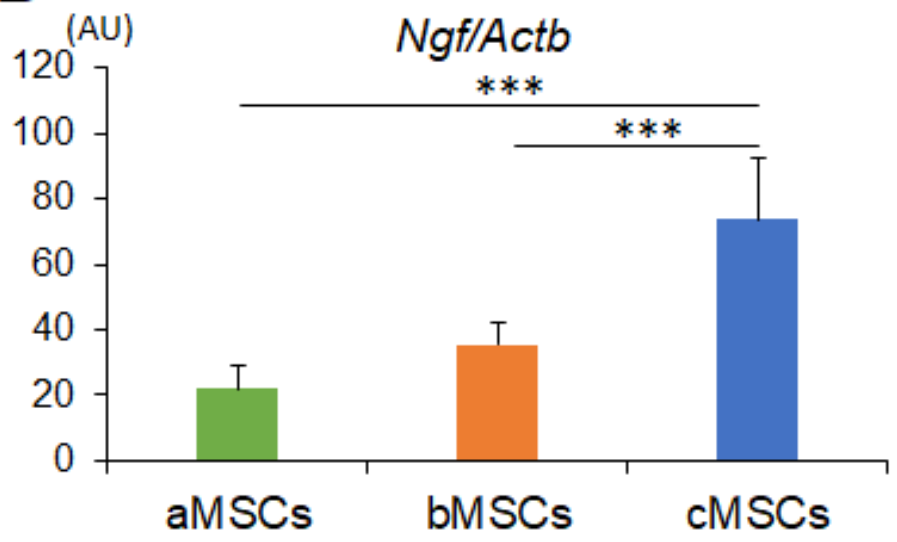

D

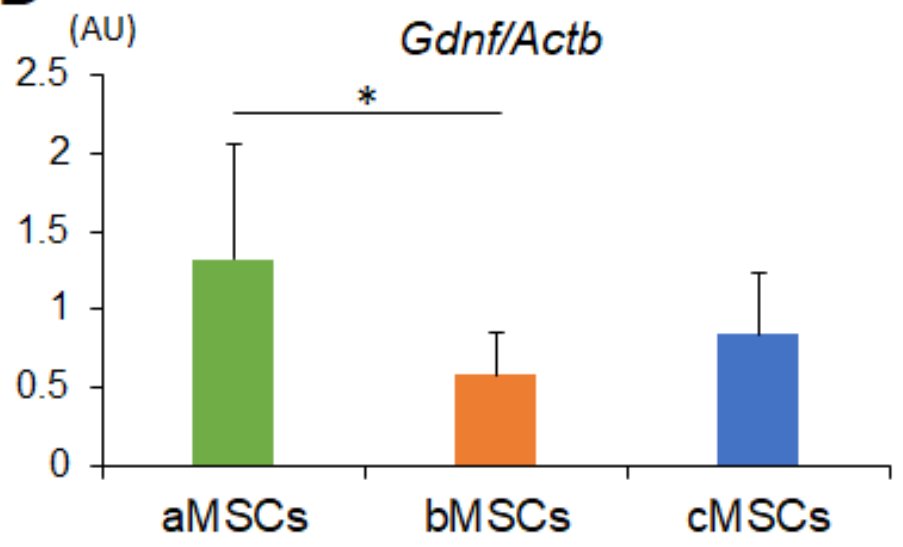

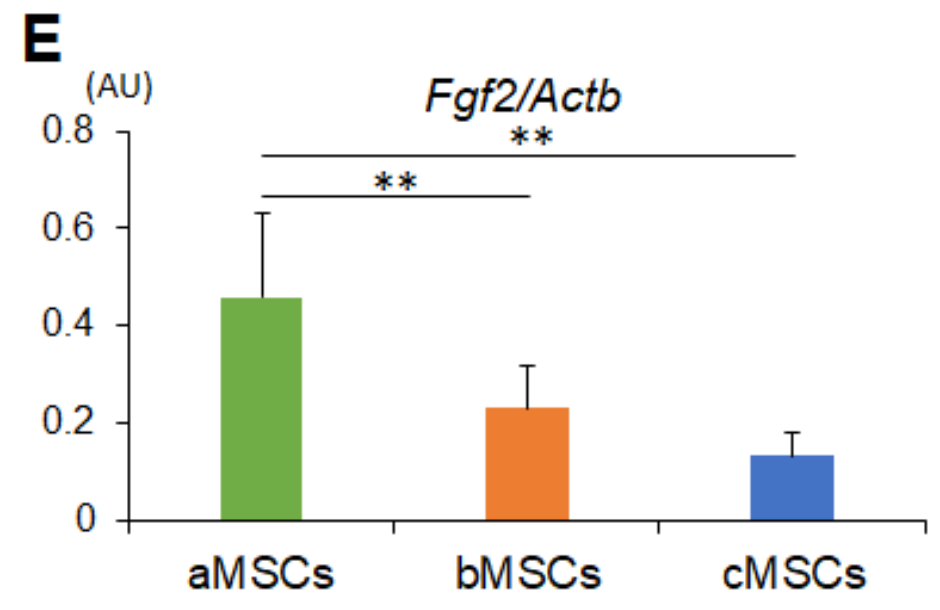

Figure 2

mRNA expressions of aMSCs, bMSCs, and cMSCs. mRNA expression levels of Bdnf (A), Ngf (B), Sort1 (Nt-3) (C), Gdnf (D), and Fgf2 (E). Bar graph of data normalized to Actb. The data are presented as the mean + SD of seven independent experiments. $n=7,{ }^{*} p<0.05$; $* \star p<0.01 ; * \star \star p<0.001$. mRNA, messenger ribonucleic acid; Bdnf, brain-derived neurotrophic factor; Ngf, nerve growth factor; Sort1 (Nt-3), sortilin 1 (neurotrophin 3); Gdnf, glial cell-derived neurotrophic factor; Fgf2, fibroblast growth factor 2; Actb, actin beta. 
A

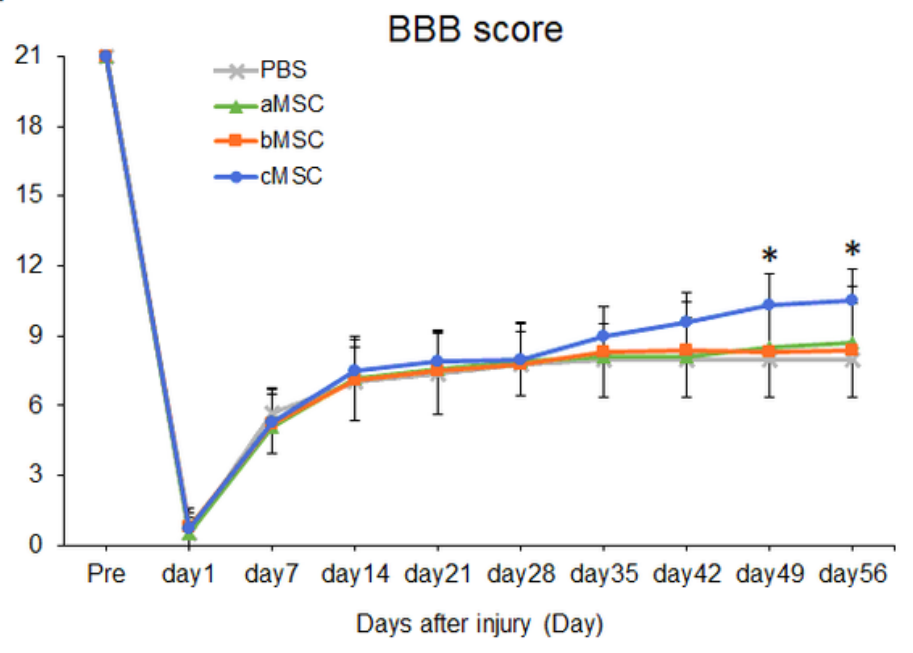

B

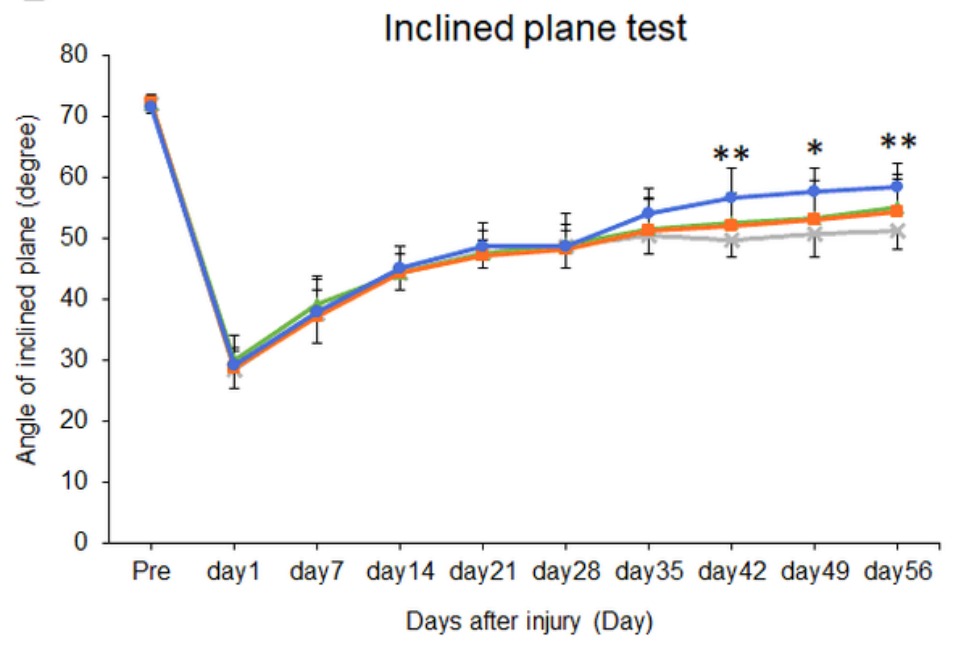

\section{Figure 3}

Motor functional improvements in chronic SCI model rats transplanted with aMSCs, bMSCs, and cMSCs. Graphs showing the BBB score $(A)$ and the inclined plane test results (B). The BBB score and the inclined plane test were measured from the time of $\mathrm{SCl}$ induction (pre) to day 56 post SCl (28 days post transplantation). The data are presented as the mean + SD of 10 independent experiments. * $p<0.05$; ** $\mathrm{p}<0.01$ (cMSC group vs. PBS group). SCl, spinal cord injury; PBS, phosphate-buffered saline. 

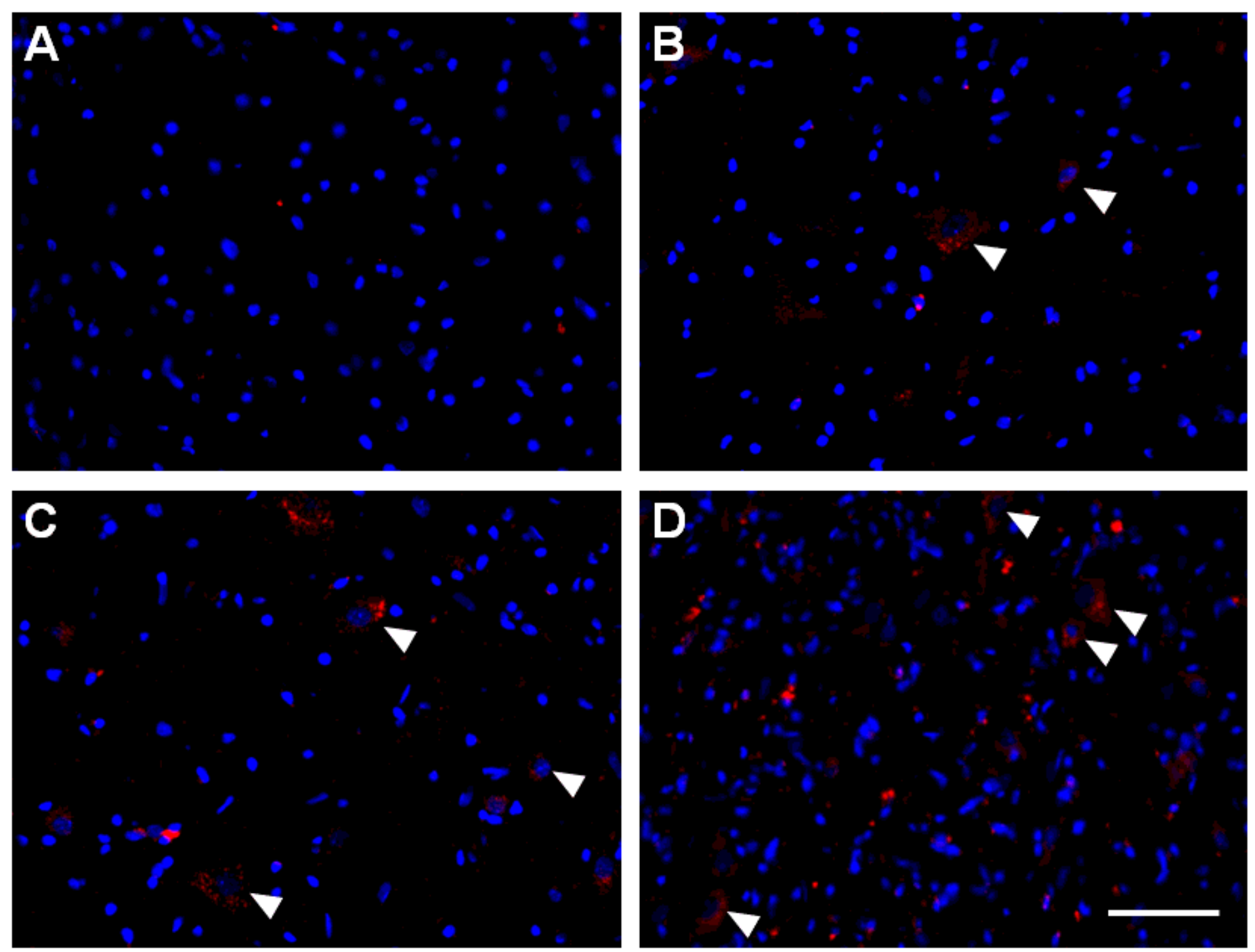

\section{Figure 4}

Detection of transplanted cells in the SC tissue of each group. Distributions of PKH26-labeled MSCs (red staining, arrowheads) in SC tissues at day 28 post transplantation. PKH26-labeled aMSCs (B), bMSCs (C), and cMSCs (D) were observed on the ventral horn of the spinal gray matter. There were no PKH26labeled cells in the PBS group (A). Nuclei were stained with DAPI (blue). Scale bars $=100 \mu \mathrm{m}$. DAPI, 4',6diamidino-2-phenylindole dihydrochloride; SC, spinal cord. 
A

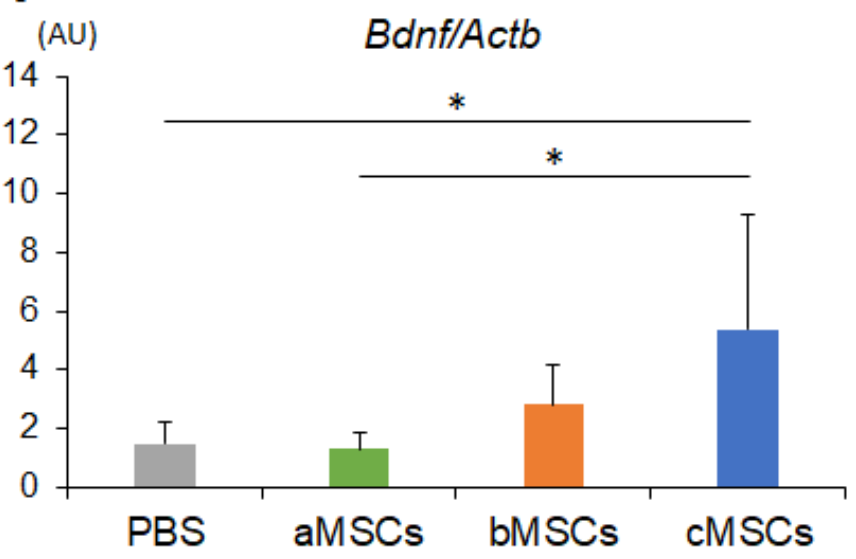

C

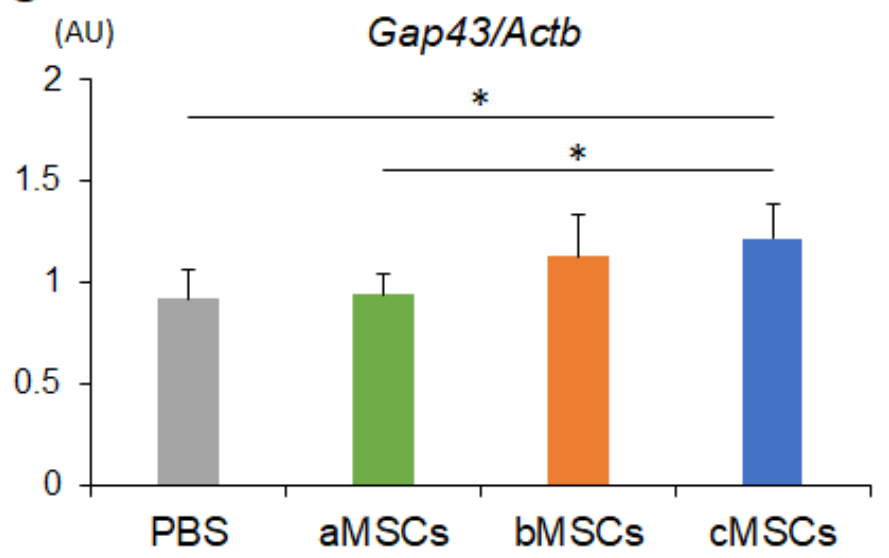

B

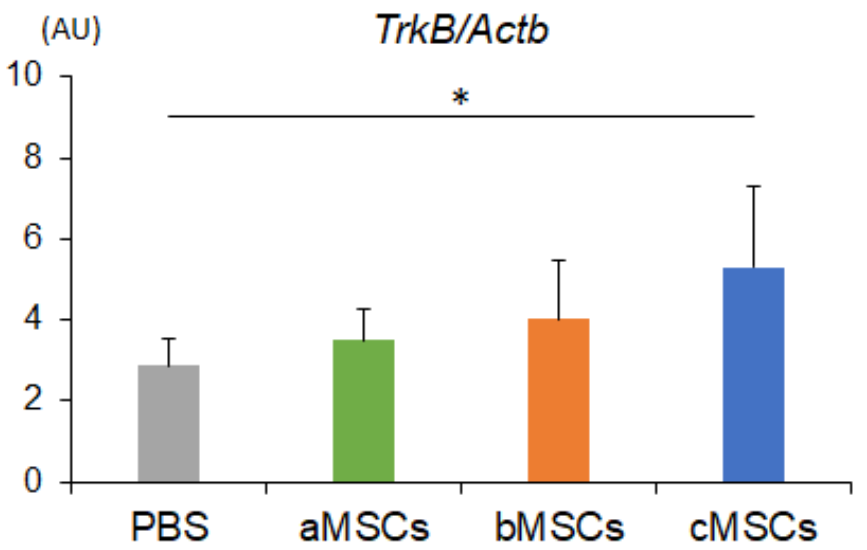

D

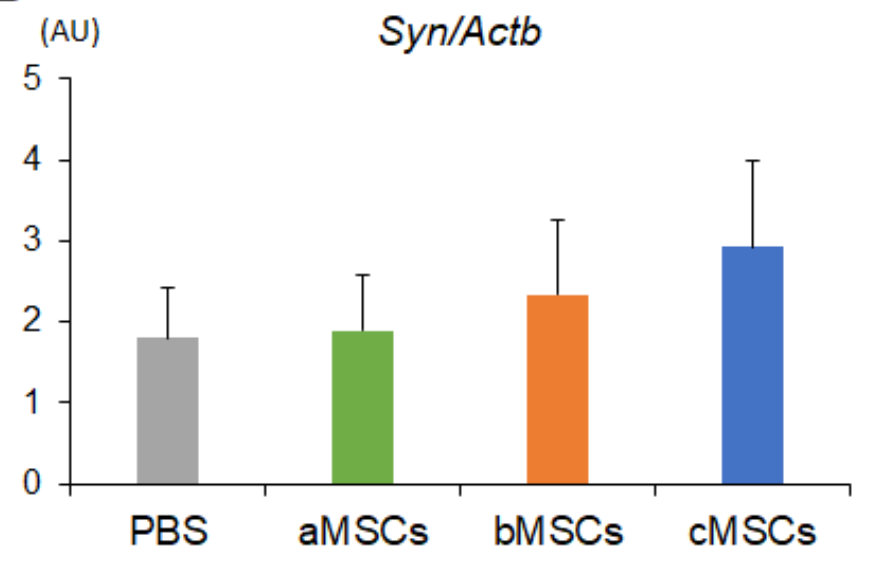

Figure 5

mRNA expression levels in injured rat SC tissues. mRNA expression levels of Bdnf (A), TrkB (B), Gap-43 (C), and Syn (D) in SC tissues at day 28 post MSC transplantation. Bar graph of data normalized to Actb. The data are presented as the mean $+S D$ of five independent experiments. ${ }^{*} p<0.05$. TrkB, tropomyosin receptor kinase B; Gap-43, growth associated protein 43; Syn, synaptophysin. 


\section{PBS aMSCs bMSCs cMSCs}
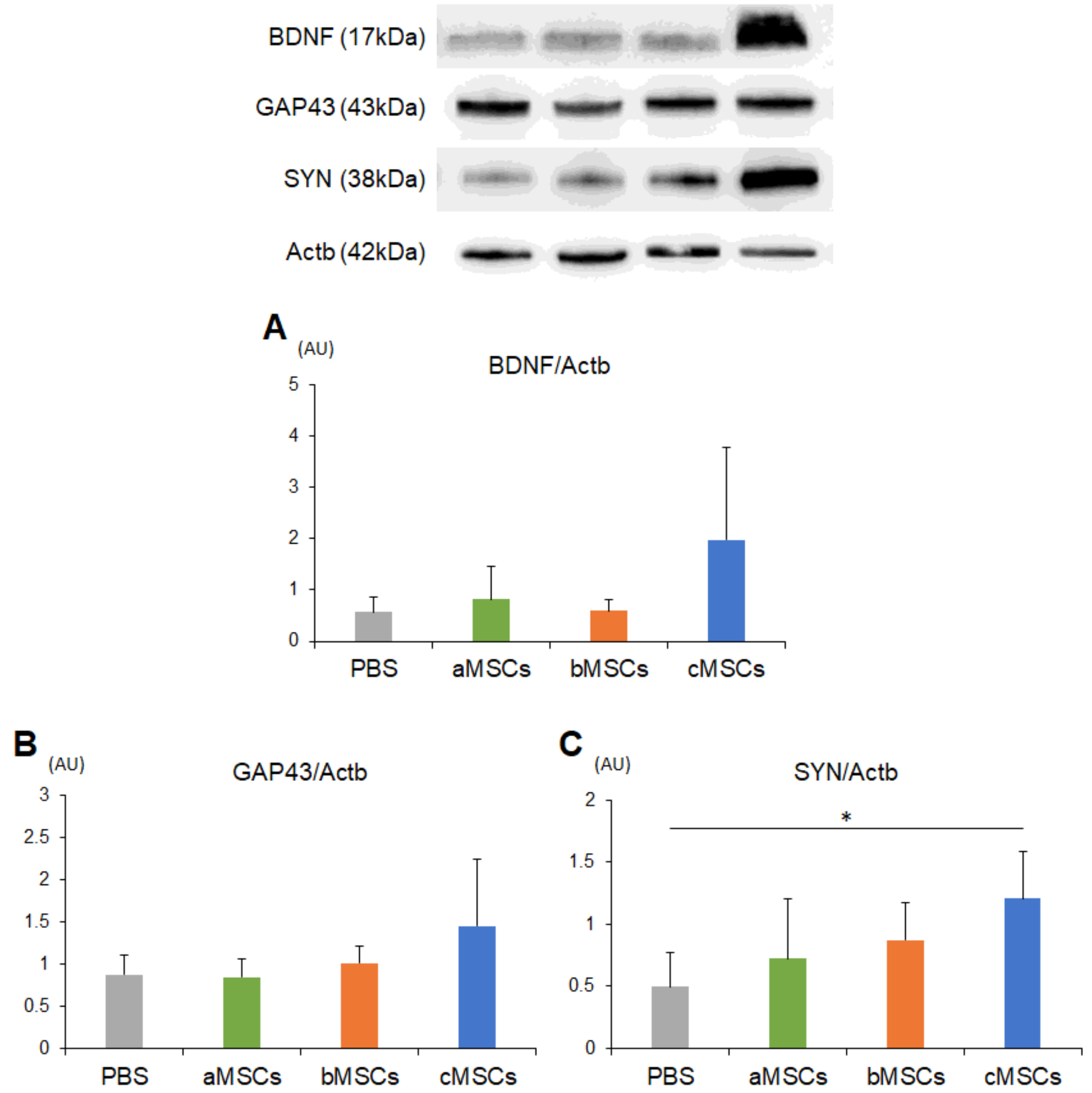

Figure 6

Protein expression levels in injured rat SC tissues. Representative electrophoresis band images and analyzed data of the protein levels of BDNF (A), GAP-43 (B), and SYN (C) in SC tissues at day 28 post MSC transplantation. Bar graph of data normalized to Actb. The data are presented as the mean + SD of five independent experiments. ${ }^{*} p<0.05$. 


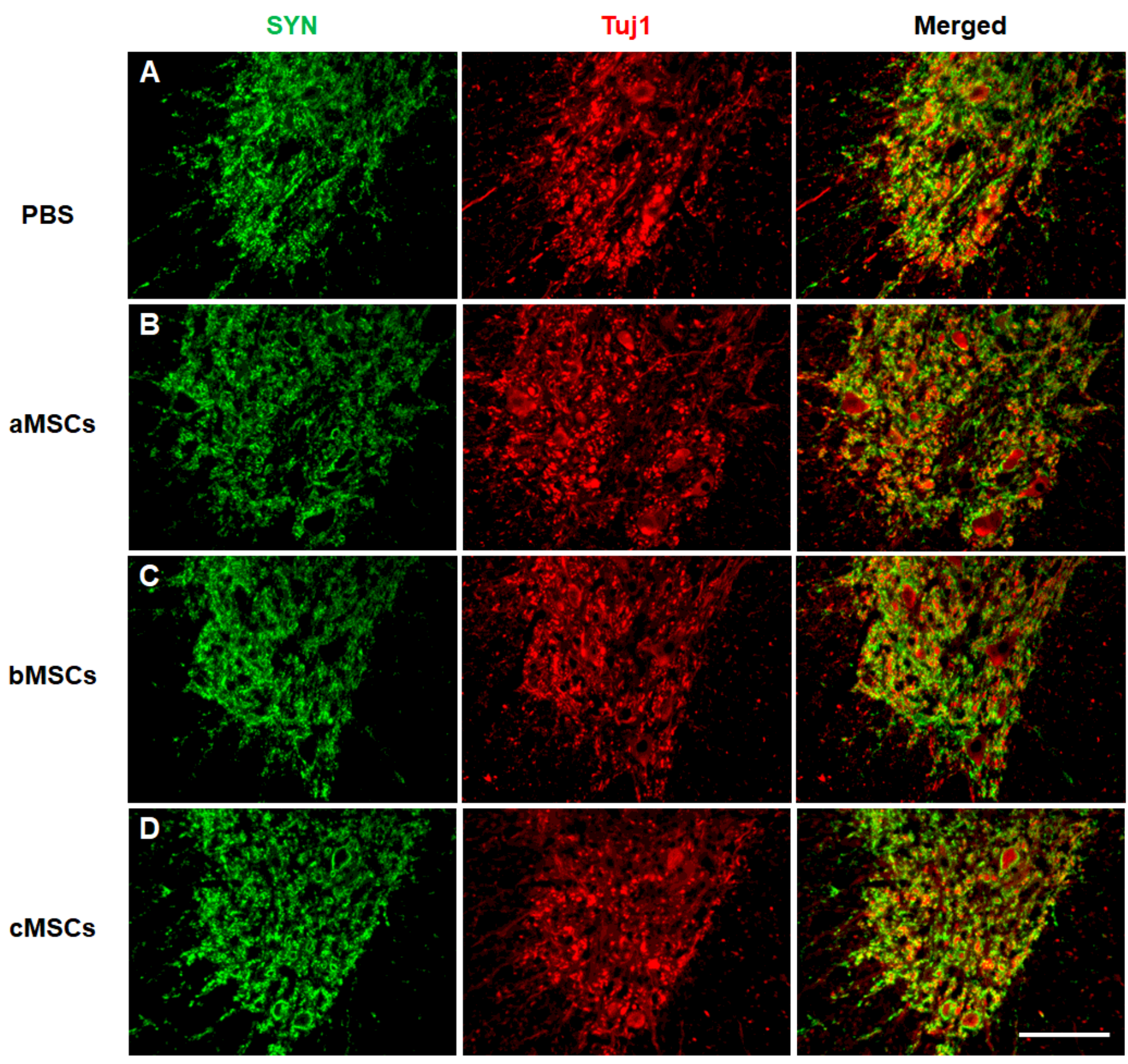

Figure 7

Photomicrographs showing immunofluorescence of SYN and Tuj1 in the ventral horn of the SC.

Photomicrographs showing immunofluorescence images of the SC tissues of the PBS group (A), aMSC group (B), bMSC group (C), and cMSC group (D) at day 28 post after each transplantation. SYN (left, green), Tuj1 (middle, red), and merged image (right). Scale bar $=100 \mu \mathrm{m}$. 
A
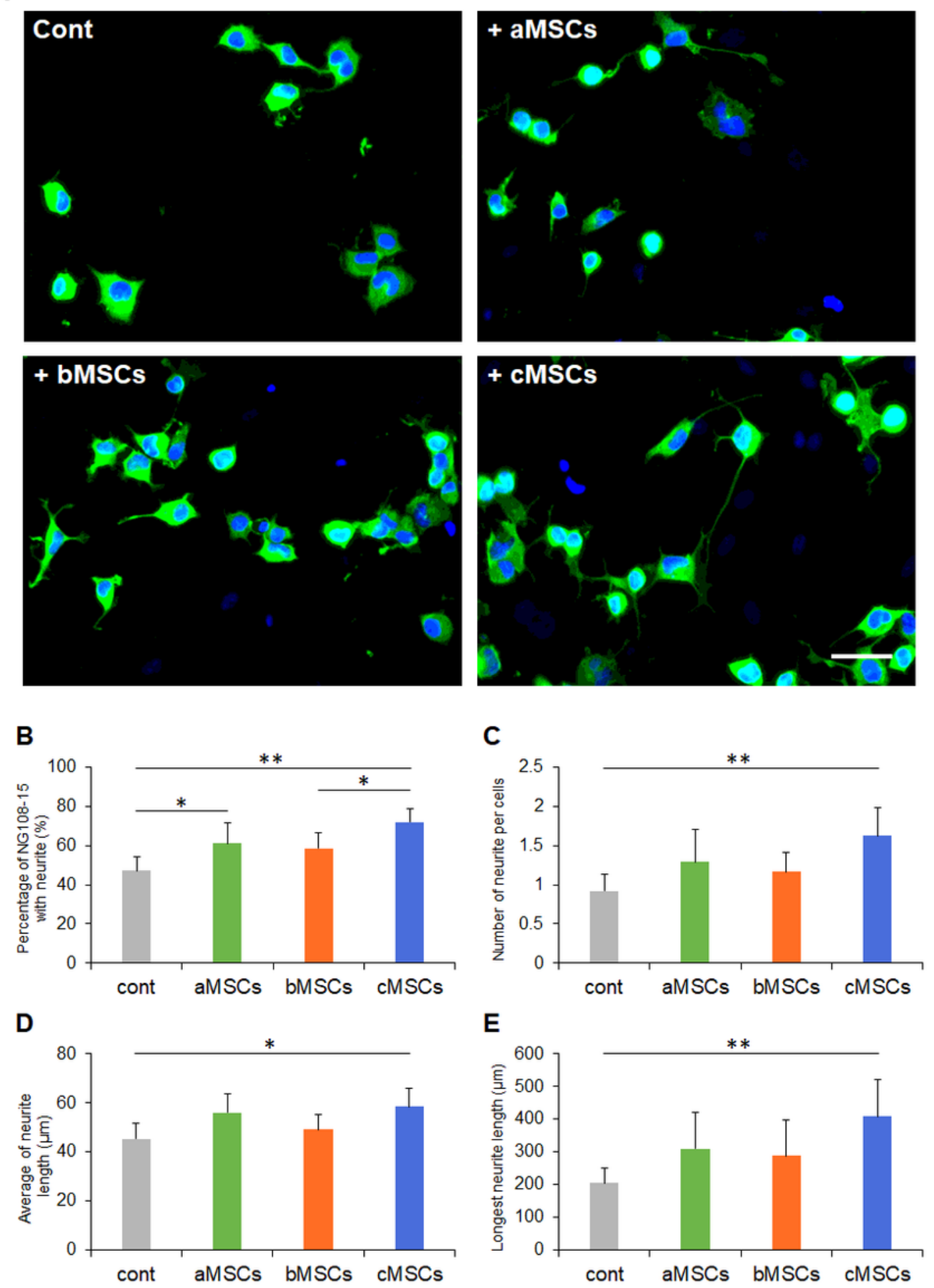

\section{Figure 8}

Neurite outgrowth analyses of NG108-15 neural cells co-cultured with aMSCs, bMSCs, and cMSCs.

NG108-15 cells were immunostained with Tuj1 Ab (green) and DAPI (blue) for neurite growth analyses. As a control, NG108-15 neural cells were cultured alone (cont). NG108-15 neural cells were co-cultured with aMSCs, bMSCs, and cMSCs (A). Scale bar $=50 \mu \mathrm{m}$. The percentage of neurite-like processes (B), number of neurites expressed per cell (C), average neurite length (D), and longest neurite in each group (E) in each 
co-culture experiment. $T$ The data are presented as the mean $+S D$ of six independent experiments. * $p<$ $0.05 ; * \star p<0.01$. 